\title{
USOS PROBABLES DE LAS MADERAS DE 20 ESPECIES DEL DEPARTAMENTO DE LORETO
}

Baluarte $\mathrm{V}$. Juan Rommel $\left({ }^{*}\right)$

A rostegui Vargas A ntonio $\left({ }^{* *}\right)$

\section{RE SU M E N}

En este artículo se da a conocer los usos probables de las maderas de veinte especies forestales de las zonas de Colonia Angamos y Jenaro Herrera (Loreto), determinados por una metodología basada en las características generales y ensayos físico mecánicos de estas especies.

L os resultados de los análisis realizados en los laboratorios de tecnología de la madera de la Universidad Nacional de la A mazonia Peruana (Iquitos), Centro Forestal XII (Pucallpa) e Instituto Nacional de Pesquisas de la A mazonia (M anaus - Brasil), se adjuntan en los cuadros del anexo.

Este trabajo constituye una primera presentación de resultados de los estudios tecnológicos de maderas de selva baja que viene ejecutando el IIAP en convenio con instituciones nacionales y extranjeras.

L as especies forestales que arrojaron resultados más satisfactorios son: favorito, carahuasca y papelillo caspi.

\section{INTRODUCCION}

El reducido número de especies forestales que utiliza la industria de transformación de la madera es causado en parte por la poca importancia que se ha venido brindando a estudios de investigación para conocer las características tecnológicas de nuevas especies, que podrían sustituir a las tradicionales, las mismas que por su excesiva tala se encuentran en peligro de extinción.

Por otro lado, la extracción selectiva de maderas está ocasionando escasez de materia prima valiosa y carestía de productos derivados, quedando cada vez más fuera del alcance de los usuarios de bajos recursos económicos.

El propósito fundamental del presente trabajo es determinar los usos pixbables de las maderas de 20 especies forestales de las zonas Colonia A ngamos y J enaro Herrera (L oreto). Para tal efecto, se ha recopilado toda la información básica generada por el proyecto" Básicos y A plicados de Maderas de Selva Baja", con estos resultados se determinó el comportamiento de las maderas al secado y trabajabilidad y posteriormente los usos probables de estas especies utilizando una metodología práctica, la misma que

\footnotetext{
* Director General de Investigación Forestal (IIAP)

A Asesor Científico DGIF (IIAP)
} 
será comprobada mediante la ejecución de los ensayos de laboratorio, de acuerdo a las normas técnicas correspondientes.

Obviamente, estos resultados constituyen un primer avance de lo que será el documento técnico final del proyecto arriba mencionado.

\section{REVISION DE LITERATURA}

Según AROSTEGUI (4), los inventarios forestales en el país se inician en la década de 1950 y durante los 40 últimos años se han realizado aproximadamente 120 estudios de inventarios y evaluaciones, que cubren una superficie aproximada de 46 '213,471 Ha., que corresponde al $63 \%$ de la extensión de la amazonía peruana. Estos estudios tienen carácter preliminar y no tienen la confiabilidad requerida para los planes de manejo y aprovechamiento de los bosques. Señala también que, como resultado de estos inventarios, se puede indicar que existen 96 especies diferentes, calificadas como de mayor abundancia, de las cuales el $70 \%$ alcanzan una identificación a nivel de especies y el $30 \%$ a nivel de familia. DANCE y OJEDA (10), agregan que estas 96 especies significan el $59 \%$ de los volúmenes encontrados en los inventarios; estos mismos autores añaden que ésto es una razón de mucha fuerza para atender prioritariamente estas especies en cuanto a estudios sobre identificación botánica, propiedades tecnológicas y usos. En cuanto a estudios tecnológicos y usos probables de la madera, AROSTEGUI (4) indica que, en total, se han estudiado en el país alrededor de 150 especies, de las cuales 90 especies (60\%) fueron estudiadas a nivel preliminar; es decir, con muestra procedentes de un solo árbol. Estos estudios comprenden las áreas de anatomía, propiedades físico - mecánicas, secado, preservación, uniones estructurales, trabajabilidad, pulpa y papel; en base a ellos, se han determinado los usos probables. Los análisis fueron realizados en los laboratorios del departamento de industrias forestales de la U niversidad N acional A graria - La M olina, Lima - Perú.

En un trabajo anterior, AROSTEGUI (1), reporta las propiedades tecnológicas de 145 especies forestales de diferentes zonas del país, en base a un sólo árbol por especie, considerándose los resultados de nivel preliminar.

En el estudio de las propiedades tecnológicas y usos de las maderas de 40 especies del Bosque $\mathrm{N}$ acional Alexander $\mathrm{V}$ on Humboldt, realizado por AROSTEGUI et al (6), se indican los usos probables de 40 especies, determinadas en base a cinco árboles por especie. Asimismo, AROSTEGUI y VALDERRAMA (7), realizan el estudio de maderas de 10 especies del área de Allpahuayo (Iquitos).

En lo que respecta al avance del proyecto "Estudio Básicos y A plicados de M aderas de Selva Baja", que se ejecuta en el marco del Convenio Binacional Peruano Brasilero, L LUN COR (11), reporta los valores promedios de las propiedades físicas de 20 especies y mecánicas de 5 especies del proyecto; asimismo, VALDERRAMA, LOUREIRO y A ROSTEGUI (15), reportan las características generales y macroscópicas de la madera de 20 especies; finalmente, AROSTEGUI (5), informa los valores promedios de las propiedades mecánicas de la madera de 15 especies restantes del mismo proyecto. Si bien es cierto que existe abundante información sobre un gran número de especies 
forestales, esta información en su mayor parte corresponde a especies procedentes del departamento de U cayali y muy poco a Loreto. En cuanto al nivel de precisión de los resultados, NOACK (12), indica que para los fines prácticos de un estudio sobre las propiedades tecnológicas, es suficiente tener $\pm 15 \%$ de precisión del valor promedio; para ello, se necesitan solamente cinco árboles seleccionados al azar, agrega también que, en la práctica, está demostrado claramente que lo más económico es tomar solamente dos muestras, dado que con solo dos muestras es posible también estimar la variación de las propiedades dentro de los árboles.

\section{METODOLOIA}

\subsection{Descripción ecológica del bosque}

Las muestras de madera proceden de las localidades de Colonia A ngamos y Jenaro Herrera: la primera ubicada en la margen izquierda del río $Y$ avarí, provincia de $M$ aynas y la segunda en la margen derecha del río U cayali, provincia de Requena, ambas en el departamento de L oreto, Perú.

L a formación vegetal, según el sistema de clasificación del TOSI (14), corresponde a la zona de vida natural bosque húmedo tropical (bhT).

\subsection{Selección e identificación de las especies}

Las 20 especies estudiadas fueron seleccionadas en base a su abundancia, posibilidad de extracción y de acuerdo a su valor comercial potencial.

La identificación en el bosque se efectuó en base a las características morfológicas y organolépticas de la especie, posteriormente fue confirmada la identificación en el Herbario A mazonense de la UNAP, en base a material botánico completo.

$33 \quad$ Muestras de maderas

En el bosque se seleccionaron 5 árboles al azar de cada especie. A simismo, de cada árbol se obtuvo 3 trozas de $3 \mathrm{~m}$. de longitud, representativas de tres niveles: una de la parte baja, otra del medio y la tercera de la parte al ta.

\section{$3.4 \quad$ Normas y M étodos}

\subsubsection{Colección de muestras de madera}

Se realizó de acuerdo a la Norma COPANT 458 (9), que establece los procedimientos a seguir para realizar la selección y colección de muestras para ensayos tecnológicos, especialmente las propiedades físicas y mecánicas de la madera, a fin de obtener resultados representativos y comparables. El procedimiento de selección y colección de 
muestras se basó en el sistema de selección al azar, de modo que, en cada etapa, cada una de las unidades componentes tuviera la misma posibilidad de ser elegida. El muestreo al azar comprendió: muestreo de las áreas, de los árboles, de las trozas dentro de cada árbol y de las viguetas o listones de cada troza.

\subsubsection{Análisis y evaluación de las características generales y propiedades físico-mecánicas de las maderas}

Todos los resultados provenientes de la información básica existente del Proyecto "Estudios Básicos y Aplicados de Maderas de Selva Baja", se analizaron y evaluaron para determinar el comportamiento de las maderas al secado y trabajabilidad.

\subsubsection{Secado y trabajabilidad de las maderas}

\subsubsection{Secado}

El comportamiento de la madera al secado se ha estimado en base a las propiedades básicas, tales como la contracción tangencial (1) y contracción radial ( $R$ ), que es el índice de estabilidad de la madera, por los cambios de humedad. El comportamiento resultante esta relacionado con los defectos (grietas, rajaduras y deformaciones) que se presentan durante el secado. Maderas de baja contracción volumétrica y relación $T / R$ bajo (cuando más se acerca a 1 ), son más estables en cuanto a sus cambios dimensionales y el comportamiento al secado es bueno, es decir, sin defectos.

\subsubsection{Trabajabilidad}

Se refiere al comportamiento de la madera al trabajo con máquinas de carpintería, factor importante en la determinación de los usos. El comportamiento a la trabajabilidad se ha estimado en base a las características y propiedades relacionadas con esta particularidad: grano (dirección de las fibras), densidad básica (peso volumen verde), dureza (resistencia que ofrece la madera a la penetración de un cuerpo $\mathrm{kglcm} 2$ ), contenido de sílice (quita filo de las sierras).

\subsubsection{Determinación de los usos probables}

Los usos probables obtenidos son el resultado de una evaluación hecha para cada especie por comparación con la tabla de requerimientos mínimos de usos y con las características de maderas comerciales de uso bien conocido, tales como cedro, caoba y tornillo.

A simismo, cada uno de los resultados de las características generales y macroscópicas, propiedades físico-mecánicas, comportamiento al secado y 
trabajabilidad, cuyos cuadros se indican en el anexo, fueron analizados y procesados de acuerdo a las recomendaciones de AROSITGUI (2).

\section{RESULTADOS Y DISCUSIONES}

4.1 Identificación taxonómica de las especies estudiadas

Las especies estudiadas se indican en el Cuadro $\mathrm{N} \cong 1$ del A nexo, en este cuadro se presentan las especies forestales en orden alfabético, según su nombre común, con su respectiva identificación taxonómica. Se estudiaron en total 45 familias, correspondientes a 18 géneros.

4.2 Clasificación de las especies estudiadas según sus propiedades físicas y mecánicas

En el Cuadro $\mathrm{N} \cong 6$, se presentan las especies estudiadas de acuerdo a los rangos de clasificación de las maderas, según sus propiedades físicas y mecánicas, recomendados por PADT REFORT (13) con algunas modificaciones, razón la cual se señala en el Cuadro $\mathrm{N}$ 5 . A continuación, se presenta un análisis de las maderas de acuerdo a los resultados que se indican en los Cuadros $N 03,4,5$ y 6 , referentes a sus propiedades físicas, mecánicas y respectiva clasificación.

\subsubsection{Propiedades físicas}

\subsubsection{Densidad básica}

Del análisis de los valores promedios señalados en el Cuadro $\mathrm{N} \cong 3$ y de acuerdo a los rangos de clasificación de las especies estudiadas (Cuadros $N$ 5 y 6), se deduce que la densidad básica varía desde 0.21 $\mathrm{g} / \mathrm{cm} 3$ (punga), clasificada como madera de densidad muy baja, hasta $0.83 \mathrm{~g} / \mathrm{cm} 3$ (parinari), que corresponde a maderas de densidad muy alta. A demás, se puede observar que el $40 \%$ de las especies estudiadas corresponden a las maderas de densidad media, el $25 \%$ de las especies se encuentran dentro del grupo de maderas cuya densidad es alta, mientras que el $20 \%$ está en el grupo de maderas cuya densidad es baja, y un 10 y $5 \%$ corresponden a los grupos de maderas cuyas densidades son muy bajas y muy altas, respectivamente. 


\subsubsection{Contracción volumétrica}

Del análisis de los valores indicados en el Cuadro $\mathrm{N} \cong 3$ yde acuerdo a la clasificación de las especies estudiadas (Cuadros $\mathrm{N} 05$ y 6 ), se deduce que la contracción volumétrica varía desde

6.80 (punga), clasificada como madera de contracción volumétrica muy baja, hasta 19.20 (shiringarana), que corresponde a madera de contracción volumétrica muy alta.

A demás, se puede observar que el $36.9 \%$ de las especies estudiadas corresponden a las maderas de densidad volumétrica baja, el $31.6 \%$ se encuentran dentro del grupo de maderas de contracción volumétrica media, el $15.8 \%$ está en el grupo de maderas de contracción volumétrica muy alta, el $10.5 \%$ está incluido en el grupo de contracción volumétrica muy baja y $15.2 \%$ está en el grupo de maderas con contracción volumétrica al ta.

\subsubsection{Relación contracción tangencial y radial (TIR)}

Del análisis de los valores indicados en el Cuadro $\mathrm{N} 03$ y de acuerdo a la clasificación de las especies estudiadas (Cuadros $\mathrm{N} 05$ y 6 ), se deduce que la relación (T/R) tiene poco rango de variación, desde 1.06 (papelillo caspi), que corresponde al grupo de maderas muy estables, hasta 2.08 (pashaco curtidor), que se encuentra en el grupo de maderas estables.

\subsection{Propiedades mecánicas}

\subsubsection{Flexión estática}

Del análisis de los valores promedios (Cuadro № 4) y de acuerdo a la clasificación de las especies estudiadas (Cuadros $\mathrm{N}$ ㅇ Sy 6), se deduce que la resistencia a la flexión estática varía desde $192 \mathrm{~kg} / \mathrm{cm} 2$ (capinurí), considerado muy bajo, hasta $1039 \mathrm{~kg} / \mathrm{cm} 2$ (yacushapana), correspondiente a una resistencia ala flexión estática muy alta. A demás, se puede observar que el $29.4 \%$ de las especies estudiadas corresponden a las maderas clasificadas como de resistencia media, el $2353 \%$ de resistencia baja, el $17.65 \%$ de resistencia alta y muy alta, respectivamente, y el $11.76 \%$ de resistencia muy baja.

\subsubsection{Compresión paralela al grano}

Del análisis de los valores promedio (Cuadro № 4) y de acuerdo a la clasificación de las especies estudiadas (Cuadro № 5 y 6), se deduce que la resistencia a la compresión paralela al grano varía desde 102 $\mathrm{kg} / \mathrm{cm} 2$ (pashaco curtidor), que corresponde a una resistencia muy baja, hasta $533 \mathrm{~kg} / \mathrm{cm} 2$ (yacushapana), clasificada como madera de resistencia muy alta. 
A demás, se puede observar que el $371.58 \%$ de las especies estudiadas están en el rango de maderas con resistencia baja ala compresión paralela al grano, el $26.31 \%$ corresponde a maderas de resistencia muy baja, el $15.79 \%$ para maderas de resistencia media y alta, respectivamente, y un $10.53 \%$ a maderas con resistencia muy alta.

\subsection{3 Comprensión perpendicular al grano}

Del análisis de los valores promedio (Cuadro N2 4) y de acuerdo a la clasificación de las especies estudiadas (Cuadros N2 y 14, e deduce que la resistencia a la compresión perpendicular al grano varía desde $17 \mathrm{kglcm} 2$ (pashaco curtidor), clasificado como madera de resistencia muy bajo, hasta $17 \mathrm{~kg} / \mathrm{cm} 2$ (pashaco curtidor). clasificada como madera de resistencia muy alta.

A simismo, se puede observar que el $37.5 \%$ de las especies estudiadas corresponden a las maderas clasificadas como de resistencia baja, el $25 \%$ de resistencia media, el $18.75 \%$ de resistencia alta, el $12.5 \%$ de resistencia muy baja y el $625 \%$ de resistencia muy alta.

\subsubsection{Cizallamiento}

Del análisis de los valores promedios (Cuadro $\mathrm{N} \cong 4$ 4) y de acuerdo a la clasificación de las especies estudiadas (Cuadros $\mathrm{N}$ ㅇ 5 y 6), se deduce que la resistencia al cizallamiento varía desde $22 \mathrm{~kg} / \mathrm{cm} 2$ (punga), considerado como muy bajo, hasta $134 \mathrm{~kg} / \mathrm{cm} 2$ (yesca caspi), correspondiente a maderas de resistencia muy al ta.

Asimismo, se observa que el $40 \%$ de las especies estudiadas corresponden a las maderas clasificadas como de resistencia alta, el $26.7 \%$ de resistencia baja, el $13.33 \%$ de resistencia media, $13.33 \%$ de resistencia muy alta y un $6.7 \%$ de resistencia muy baja.

\subsubsection{Dureza}

Luego del análisis de los valores indicados en el Cuadro $\mathrm{N} N 4$ y en base a la clasificación de las especies estudiadas (Cuadros $\mathrm{N} 05$ y 6), se deduce que la resistencia ala dureza en los lados extremos varía entre $32 \mathrm{~kg} / \mathrm{cm} 2$ y $68 \mathrm{~kg} / \mathrm{cm} 2$, ambos valores correspondientes a la especie ponga, considerado como muy baja, siendo de hasta 865 $\mathrm{kg} / \mathrm{cm} 2$ y854 kg/cm2, respectivamente para la especie denominada quillobordón, considerado como muy alto.

Del análisis en conjunto de los valores indicados en el Cuadro № 4 y de los rangos de clasificación de cada una de las propiedades mecánicas (Cuadro № 5), se deduce que la menor 
resistencia mecánica corresponde a las maderas de muy baja densidad (punga y huamansamana); contrariamente a esto, la mayor resistencia mecánica corresponde a maderas de alta densidad, como el quillobordón y yacushapana; sin embargo, se considera que se requieren aún otros ensayos mecánicos complementarios para obtener resultados concluyentes.

\subsection{Comportamiento al secado y trabajabilidad}

En el Cuadro $N$ N 7, se presentan los valores cualitativos correspondientes al secado y trabajabilidad de la madera de las 20 especies estudiadas. Para este caso, se ha tomado en cuenta los criterios de clasificación utilizados por AROSTEGUI (2).

\subsubsection{Secado}

Del Cuadro $\mathrm{N}$ 7, se deduce que las maderas según el comportamiento al secado, se clasifican en tres grupos:

\section{GRUPO I: Comportamiento regular}

Maderas con defectos, grietas, rajaduras y deformaciones moderadas: quillobordón, parinari, capinurí, shiringarana, goma pashaco, pashaco curtidor, yesca caspi y aguano cumala.

\section{GRUPO II: Comportamiento bueno}

Maderas con defectos, grietas y deformaciones leves: moena amarilla, ana caspi, añuje moena, punga, yacushapana, carahuasca, huamansamana, rifan blanco y favorito.

\section{GRUPO III: Comportamiento muy bueno}

Maderas sin defectos, grietas y deformaciones: caucho masha y papelillo caspi.

Del análisis de los grupos de clasificación indicados, se observa que el $42.10 \%$ de las especies estudiadas corresponden a maderas con comportamiento regular al secado, el $47.37 \%$ corresponden a maderas de comportamiento bueno y un $10.53 \%$ a maderas de comportamiento al secado muy bueno.

\subsection{Trabajabilidad}

Del Cuadro № 7, se deduce que las maderas de las especies estudiadas, según el comportamiento al trabajo con máquinas de carpintería (Trabajabilidad), se clasifican en cuatro grupos: 
GRUPO I: Comportamiento muy bueno

M aderas con densidad media, grano recto y dureza baja: favorito.

GRUPO II: Comportamiento bueno

$M$ aderas con densidad muy baja a media, grano recto, dureza muy baja a baja: punga, papelillo caspi, carahuasca, huamansamana y aguano cumala.

GRUPO III: Comportamiento regular

Maderas con densidad baja a alta, grano redo, entre cruzado a oblicuo, dureza muy baja a media: moena amarilla, añuje moena, caucho masha, loro shungo, capinurí, rifari blanco, shiringarana, goma pashaco y pashaco curtidor.

GRUPO IV: Comportamiento malo

$M$ aderas con densidad alta a muy alta, grano recto ha entrecruzado, contenido de sílice, dureza baja, alta o muy alta: quillobordón, yacushapana, parinari colorado, ana caspi y yesca caspi.

Del análisis de los grupos de clasificación indicados, se observa que el $5 \%$ de las especies estudiadas corresponden a maderas de comportamiento muy bueno a la trabajabilidad, el $25 \%$ corresponden a maderas de comportamiento bueno, el $45 \%$ a maderas de comportamiento regular y el $25 \%$ a maderas de comportamiento malo a la trabajabilidad.

\subsection{Usos probables}

L as características cualitativas y propiedades relacionadas con el uso: densidad básica, resistencia mecánica, secado, trabajabilidad y veteado de la madera se presentan para cada una de las especies estudiadas en el Cuadro $\mathrm{N} 08$.

La combinación de estas características y propiedades sirven para la determinación de los usos. Para seleccionar la mejor madera para una aplicación particular, es necesario saber los requerimientos mínimos de uso y conocer las características y propiedades de las maderas.

En base a las características y propiedades analizadas y evaluadas y tomando en cuenta los requisitos mínimos de uso y de acuerdo a la comparación con maderas comerciales de uso bien conocido (cedro, caoba y tornillo), sc determinaron los usos probables, los que se presentan en el Cuadro $\mathrm{N} 09$. 


\subsubsection{Construcción de viviendas}

\subsubsection{Estructuras:}

Vigas, columnas, viguetas, y otros elementos estructurales: papelillo caspi, carahuasca, shiringarana, rifan blanco, añuje moena y moena amarilla.

\subsubsection{Carpintería de obra:}

Puertas, ventanas y zócalos: favorito, moena amarilla, carahuasca, papelillo caspi, shiringarana, caucho masha, loro shungo, aguano cumala y añuje moena.

\subsubsection{Parquet:}

A na caspi, quillobordón, paninani colorado, yesca caspi y yacushapana.

\subsubsection{Encofrados:}

A guano cumala, añuje moena, capinurí, carahuasca, caucho masha, goma pashaco, loro shungo, pashaco curtidor, punga y huamansamana.

\subsection{Construcción pesada}

Durmientes, pilotes, puentes y puntales (para minas)

\subsubsection{Durmientes:}

Y esca caspi, yacushapana, ana caspi, shiringarana, rifan blanco, añuje moena y quillobordón.

\subsubsection{Pilotes, puentes y puntales:}

Y esca caspi, yacushapana, ana caspi, parinani colorado y quillobordón.

\subsubsection{Mobiliario en general:}

Bancos, mesas, reposteros, armarios y carpetas: aguano cumala, añuje moena, carahuasca, favorito, moena amarilla y papelillo caspi.

\subsubsection{Chapas (compensados y decorativos):}

Capinurí, caucho masha, punga, favorito, yacushapana y yesca caspl.

\subsubsection{Mango de herramientas:}

A ñuje moena, rifan blanco, shiringarana y yesca caspi.

\subsubsection{Carrocería:}

Y acushapana y ana caspi. 


\subsubsection{Cajonería liviana:}

Caucho masha, goma pashaco, pashaco curtidor, huamansamana, loro shungo y punga.

4.49 E banistería:

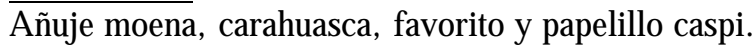

\subsubsection{Instrumentos (científicos y profesionales)}

Favorito, carahuasca y papelillo caspi.

\subsubsection{Piezas torneadas:}

Ana caspi, añuje moena, favorito, moena amarilla, papelillo caspi, parinari colorado, quillobordón, rifan blanco, shiringarana, yacushapana y yesca caspi.

\section{BIBLIOGRAFIA}

1. AROSTEGUI, A. 1975. Estudio tecnológico de maderas del Perú (Zona Pucallpa) Vol. 1; características tecnológicas y usos de la madera de 145 especies del país.

M inisterio de A gricultura, Universidad Nacional Agraria - La M olina, Lima Perú. 483 pág.

2. AROSTEGUI, A. 1975. Estudio tecnológico de maderas del Perú (Zona Pucallpa) V ol. II. M étodos y especificaciones empleados en los estudios tecnológicos de maderas. M inisterio de A gricultura, U niversidad Nacional A graria- La M olina. Lima - Perú. 104 pág.

3. AROSTEGUI, A. 1979. Estudio integral de la madera para construcción. M inisterio de A gricultura y Alimentación, U niversidad Nacional Agraria - La Molina. Lima - Perú. 166 pág.

4. AROSTEGUI, A. 1986. Expediente técnico del proyecto "Estudios Básicos y Aplicados de Maderas de Selva Baja." Instituto de Investigaciones de la A mazonía Peruana. Iquitos - Perú. 23 pág.

5. AROSTEGUI, A. 1987. Informe técnico semestral. Proyecto "Estudios Básicos y A plicados de la M adera, estudio propiedades físicas y mecánicas de maderas de Selva baja. Instituto de Investigaciones de la A mazonía Peruana (IIAP). Centro Forestal XII (CENFOR XII). Pucallpa - Perú. 9 pág. 
6. AROSTEgUI, A. et al 1975. Estudio tecnológico de maderas del Perú (Zona Pucallpa)

Vol. III; Características tecnológicas de la madera de 40 especies del Bosque Nacional A lexander V on Humboldt, M inisterio de A gricultura, U niversidad Nacional A graria - La M olina, Lima - Perú. 171 pág.

7. AROSTEGUI, A. y VALDERRAMA, H. 1986. Usos de las maderas del bosque húmedo tropical "Allpahuayo" - I quitos. Serie Investigaciones Tecnológicas. A ño, № 05. Instituto de Investigaciones de la Amazonía Peruana, Universidad Nacional de la Amazonía Peruana. Iquitos - Perú. 27 pág.

8. BALUARTE, J; AROSTEGUI, A. y REVOREDO, N. 1986. Informe técnico final Proyecto "U sos de las $M$ aderas del Bosque Húmedo Tropical (bh-T) Colonia A ngamos (río Y avarí) y Jenaro Herrera"; Sub Proyecto "Identificación y colección de maderas de especies forestales de Selva baja", IIA P -Región A graria XXII - Loreto, Iquitos - Perú, 112 pág.

9. COPANT. 1972. COMISION PANAMERICANA DE NORMAS TECNICAS; "Maderas, selección y colección de muestras" COPANT 458: 1 -11. Lima, 11 pág.

10. DANCE, J. y OJEDA W. 1979. Evaluación de los recursos forestales del trópico peruano. Universidad Nacional Agraria - La M olina, Departamento de M anejo Forestal. Lima Perú. 119 pág.

11. LLUNCOR, D. 1986, Informe técnico correspondiente al año 1985 yprimertrimestre1986. Proyecto "Usos de las Maderas del Bosque Húmedo Tropical Colonia Angamos (río Y avar9 y Jenaro Herrera', Sub - proyecto "Propiedades físicas y mecánicas de la madera de veinte especies forestales de la zona Colonia A ngamos (río Y avarí y Jenaro Herrera". Instituto de Investigaciones de la

A mazonia Peruana (IIA P), Centro Forestal XII (CENFOR XII) - Pucallpa - Perú. 12 pág.

12. NOACK DETLEF. Evaluación de propiedades de maderas tropicales. Hamburgo - A lemania.

13. PADT - REFORT. Tablas de propiedades físicas y mecánicas de la madera de veinte (20) especies del Perú. Grupo A ndino - J unta del A cuerdo de Cartagena. Lima - Perú. 53 pág.

14. TOSI, J. Jr. 1960 Zonas de vida natural en el Perú. Lima IICA-OEA. Zona andina. Lima Perú. (Boletín técnico № 3). 27 pág.

15. VALDERRAMA, H.; LOUREIRO, A. y AROSI'EGUI, A. 1986. Informe técnico correspondiente al año 1985. Proyecto " $U$ sos de las $M$ aderas del Bosque Húmedo Tropical Colonia Angamos (río Y avarí) y Jenaro Herrera"; Sub-proyecto "Estructura anatómica y clave de identificación de veinte especies forestales de la zona de Colonia Angamos (río Y avarí) y Jenaro Herrera'. Instituto de Investigaciones de la A mazonía Peruana (IIA P), Universidad Nacional de la A mazonía Peruana (UN AP). Iquitos - Perú. 109 pág. 


\section{CUADRO N²}

RELACION DE ESPECIES ESTUDLADAS DE COLONIA ANGAMOS Y JENARO HERRERA (LORETO)

$\mathrm{N}^{2}$ Nombre Común NOMBRE CIENTIFICO

01 Aguano cumala

02 Ana caspi

03 Afiuje moena

04 Capinurí

05 Carahuasca

06 Caucho masha

07 Favorito

08 Goma pashaco

09 Huamansamana

10 Loro shungo

11 Moena amarilla

12 Papelillo caspi

13 Parinari colorado

14 Pashaco curtidor

15 Punga

16 Quillobordón

17 Rifari blanco

18 Shiringarana

19 Yacushapana

20 Yesca caspi
Virola albidiflora Ducke

Apuleia molaris Spruce ex Benth.

Anaueria sp.

Maquira coreacea (Karsten) C.C. Berg

Guatteria hyposericea Diels

Brosimum parinarioides Ducke subsp.

amplicoma (Ducke) C.C. Berg

Osteophloeum platyspermum (A. DC.)

Warburg

Parkia igneiflora Ducke

Iacaranda copaia (Aubl.) D. Don

subsp. spectabilis (Mart. ex DC) A.

Gentry

Brosimum potabile Ducke

Aniba puchury-minor (C. Mart.) Mez.

Cariniana decandra Ducke

Licania micrantha Miquel

Parkia multijuga Benth.

Bombax munguuba Mart. y Succ.

Aspidosperma marcgravianum

Woodson

Miconia poeppigii Triana

Micranda spruceana (Baill.) R.E.

Schultes

Buchenavia capitata (Vahl) Eichl.

Qualea paraensis Ducke
FAMILIA

Myristicaceae

Caesalpinaceae

Lauraceae

Moraceae

Annonaceae

Moraceae

Myristicaceae

Mimosaceae

Bignoniaceae

Moraceae

Lauraceae

Lecythidaceae

Crisobalanaceae

Mimosaceae

Bombacaceae

Apocynaceae

Melastomaceae

Euphorbiaceae

Combretaceae

Vochysiaceae

FUENTE: BALUARTE, AROSTEGUI y REVOREDO (8) 


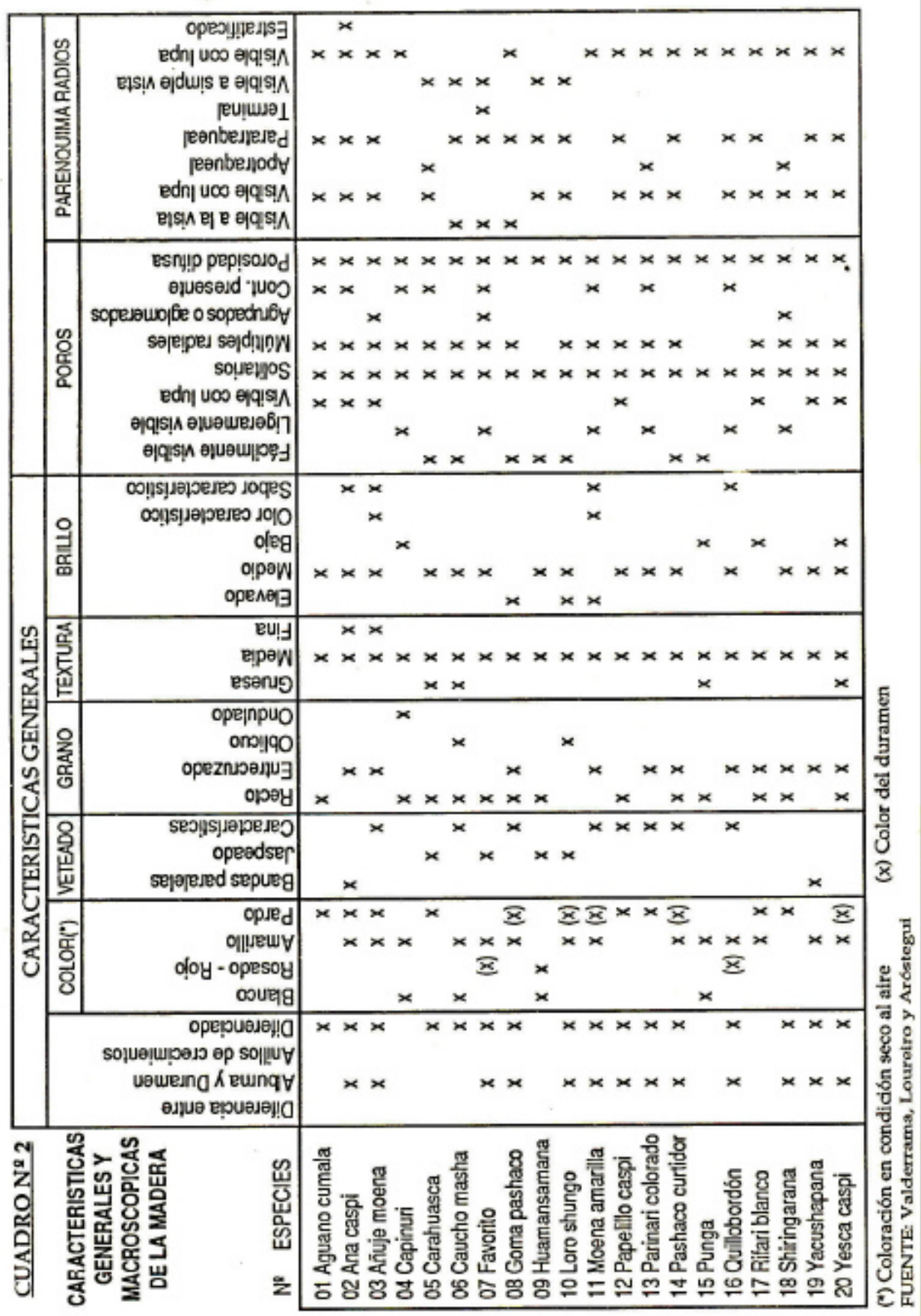




\section{CUADRO № 3}

PROPIEDADES FISICAS DE LAS 20 ESPE CIES

\begin{tabular}{|l|l|r|r|r|r|r|r|}
\hline $\mathrm{N} 0$ & \multicolumn{1}{|c|}{$\begin{array}{c}\text { NOM BRE } \\
\text { COMUN }\end{array}$} & \multicolumn{1}{c|}{$\begin{array}{c}\mathrm{C} . \mathrm{H} \\
\%\end{array}$} & $\begin{array}{c}\mathrm{D} . \mathrm{B} \\
\mathrm{G} / \mathrm{cm}^{3}\end{array}$ & \multicolumn{1}{c|}{$\begin{array}{c}\mathrm{C} . \mathrm{R} \\
\%\end{array}$} & \multicolumn{1}{c|}{$\begin{array}{c}\mathrm{C} . \mathrm{T} \\
\%\end{array}$} & \multicolumn{1}{c|}{$\mathrm{C} . \mathrm{V}$} & $\mathrm{T} / \mathrm{R}$ \\
\hline 01 & A guano cumala & 177.47 & 0.35 & 6.00 & 9.20 & 14.73 & 1.53 \\
02 & A na caspi & 32.27 & 0.70 & 4.20 & 6.40 & 10.60 & 1.52 \\
03 & A ñuje moena & 72.07 & 0.60 & 5.67 & 7.67 & 13.00 & 1.34 \\
04 & Capinuri & 153.87 & 0.37 & 3.27 & 6.07 & 9.53 & 1.85 \\
05 & Carahuasca & 92.67 & 0.41 & 4.87 & 7.27 & 12.07 & 1.49 \\
06 & Caucho masha & 146.67 & 0.42 & 4.00 & 4.53 & 8.53 & 1.13 \\
07 & Favorito & 67.93 & 0.49 & 4.67 & 6.60 & 11.13 & 1.41 \\
08 & Goma pashaco & 113.93 & 0.34 & 3.07 & 6.20 & 9.33 & 2.01 \\
09 & Huamansamana & 280.53 & 0.25 & 4.33 & 6.13 & 10.27 & 1.41 \\
10 & Loro shungo & 100.33 & 0.49 & 4.40 & - & - & - \\
11 & M oena amarilla & 56.27 & 0.52 & 3.80 & 5.73 & 9.53 & 1.50 \\
12 & Papelillo caspi & 77.20 & 0.51 & 4.80 & 5.13 & 9.93 & 1.06 \\
13 & Parinari colorado & 44.67 & 0.83 & 8.00 & 11.40 & 18.80 & 1.42 \\
14 & Pashaco curtidor & 196.60 & 0.31 & 3.87 & 8.07 & 11.94 & 2.08 \\
15 & Punga & 247.00 & 0.21 & 2.27 & 4.53 & 6.80 & 1.99 \\
16 & Quillobordón & 38.73 & 0.78 & 6.73 & 9.20 & 15.33 & 1.36 \\
17 & Rifari blanco & 81.27 & 0.58 & 3.27 & 6.07 & 9.34 & 1.85 \\
18 & Shiringarana & 62.23 & 0.66 & 8.60 & 11.27 & 19.87 & 1.31 \\
19 & Y acushapana & 44.00 & 0.78 & 5.07 & 6.66 & 11.73 & 1.31 \\
20 & Y esca caspi & 57.60 & 0.63 & 4.20 & 8.53 & 12.73 & 2.03 \\
& & & & & & & \\
\hline
\end{tabular}

Fuente: LLUNCOR, D. (11) 


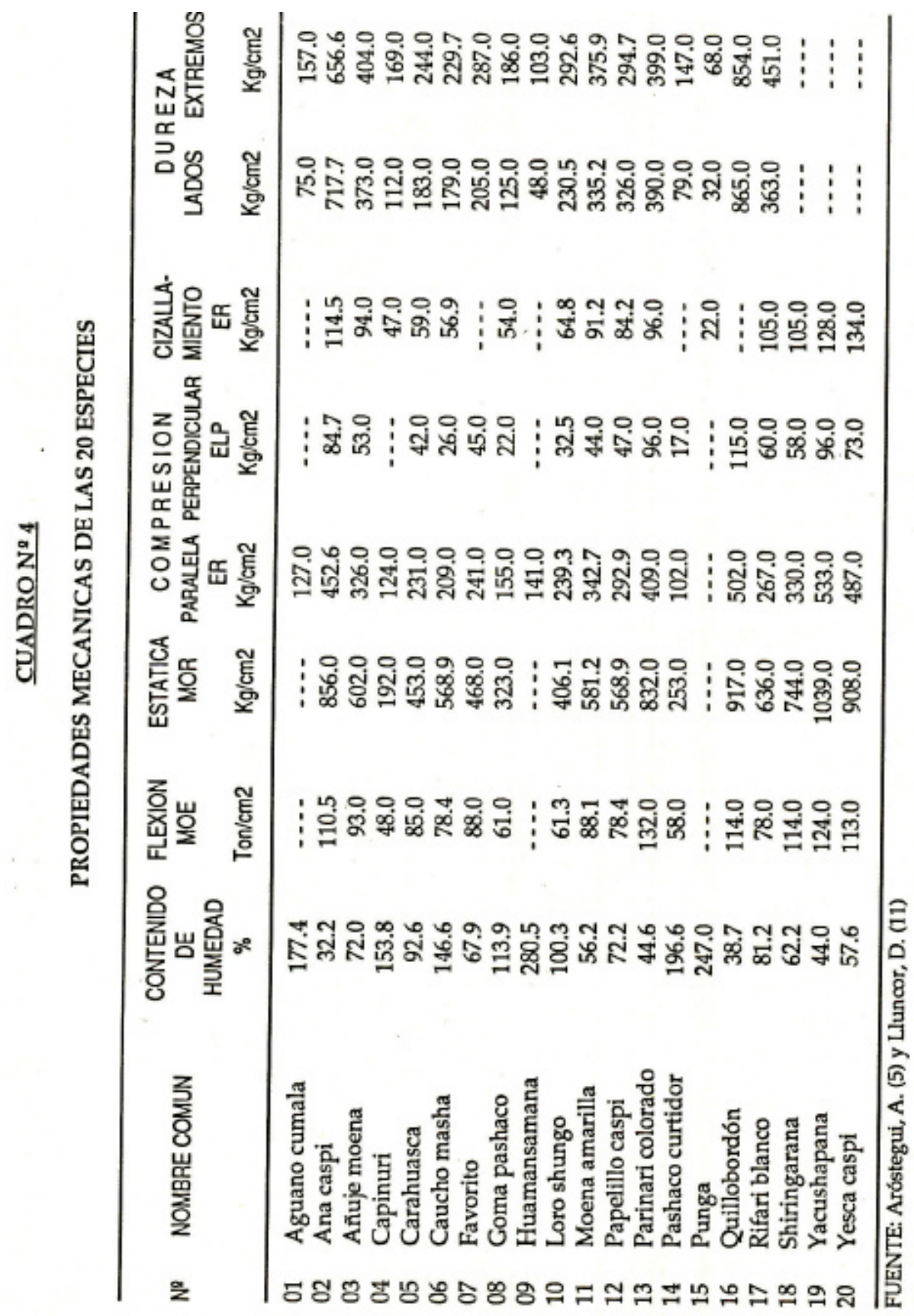




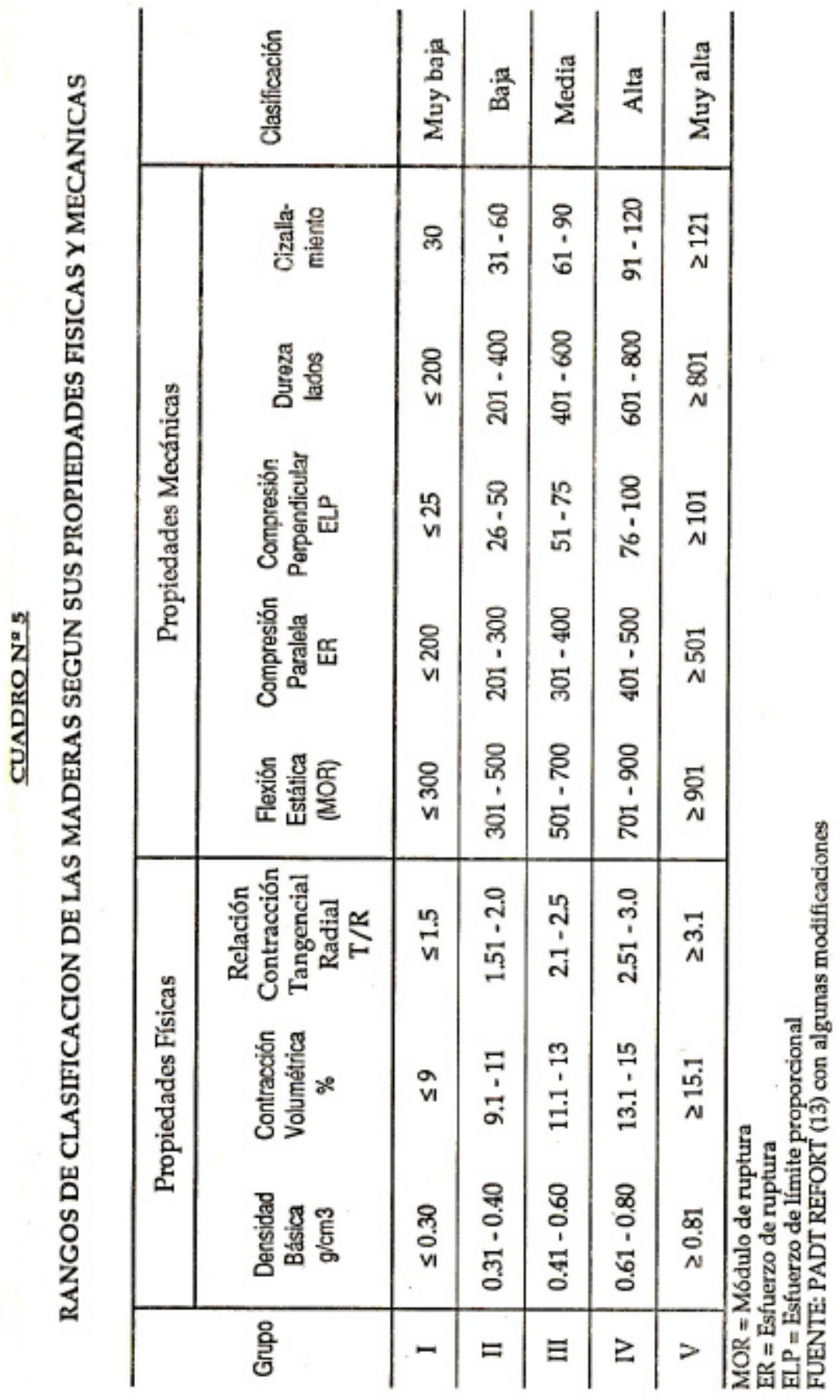




\section{CUADRON6}

\section{CLASIFICACION DE LAS ESPECIES ESTUDIADAS SEGUN SUS PROPIEDADES FISICAS Y MECANICAS}

\begin{tabular}{|c|c|c|c|c|c|c|c|c|}
\hline \multirow[b]{2}{*}{ Nombre Común } & \multicolumn{3}{|c|}{ Propiedades fisicas } & \multicolumn{5}{|c|}{ Propieclades Mecánicas } \\
\hline & $\begin{array}{c}\text { Densidad } \\
\text { Búsica }\end{array}$ & $\begin{array}{c}\text { Contrac. } \\
\text { Volu. }\end{array}$ & $\begin{array}{c}\text { Relación } \\
\text { T/R }\end{array}$ & $\begin{array}{c}\text { Fexión } \\
\text { Estática } \\
\text { MOR }\end{array}$ & $\begin{array}{c}\text { Comp. } \\
\text { Paralela } \\
\text { ER }\end{array}$ & $\begin{array}{c}\text { Comp. } \\
\text { Perpen. } \\
\text { ELP }\end{array}$ & $\begin{array}{l}\text { Dureza } \\
\text { Lados }\end{array}$ & $\begin{array}{l}\text { Cizalla- } \\
\text { miento }\end{array}$ \\
\hline
\end{tabular}

\begin{tabular}{llllllllll}
01 & Aguano cumala & BA & ME & BA & -- & MB & .- & MB & -- \\
\hline 02 & Ana caspi & AL & BA & BA & AL & AL & AL & AL & AL \\
\hline 03 & Affuje moena & ME & ME & MB & ME & ME & ME & BA & AL
\end{tabular}

\begin{tabular}{llllllllll}
04 & Capinurí & BA & BA & BA & MB & MB & $\ldots$ & MB & BA \\
\hline
\end{tabular}

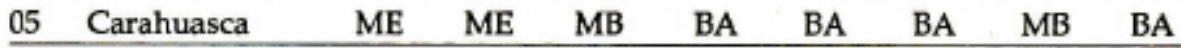

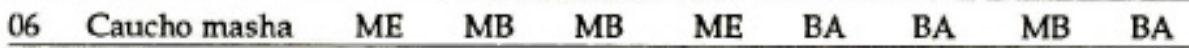

\begin{tabular}{llllllllll}
07 & Favorito & ME & ME & MB & BA & BA & BA & BA &.- \\
\hline 08 & Coma pashaco & BA & BA & BA & BA & MB & MB & MB & BA
\end{tabular}

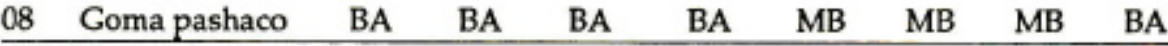

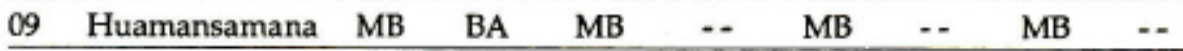

\begin{tabular}{llllllllll}
\hline 10 & Loro shungo & ME & -- & -- & BA & BA & BA & BA & ME \\
\hline 11 & Moena amarilla & ME & BA & MB & ME & ME & BA & BA & AL \\
\hline 12 & Papelillo caspi & ME & BA & MB & ME & BA & BA & BA & ME
\end{tabular}

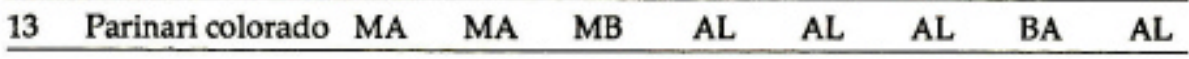

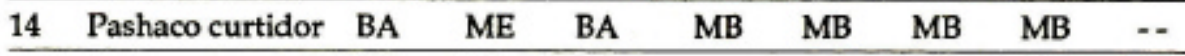

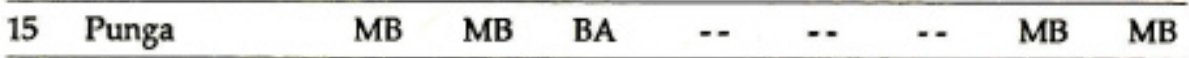

\begin{tabular}{llllllllll}
\hline 16 & Quillobordón & AL & MA & MB & MA & MA & MA & MA & -- \\
\hline
\end{tabular}

\begin{tabular}{llllllllll}
\hline 17 & Rifari blanco & ME & BA & BA & ME & BA & ME & BA & AL \\
\hline 18 & Shiringarana & AL & MA & MB & AL & ME & ME & $\ldots$ & AL \\
\hline 19 & Yacushapana & AL & ME & MB & MA & MA & AL & $\ldots$ & MA \\
\hline 20 & Yesca caspi & AL & ME & BA & MA & AL & ME & $\ldots$ & MA
\end{tabular}

$T / R=$ Tangencial $/$ Radial

MOR = Módulo de ruptura

$E R$ = Esfuerzo de ruptura

ELP = Esfuerzo al límite proporcional

$\mathrm{MB}=$ Muy Baa; $\mathrm{BA}=$ Baja; $\mathrm{ME}=$ Media; $\mathrm{AL}=\mathrm{Alta} ; \mathrm{MA}=$ Muy alta 


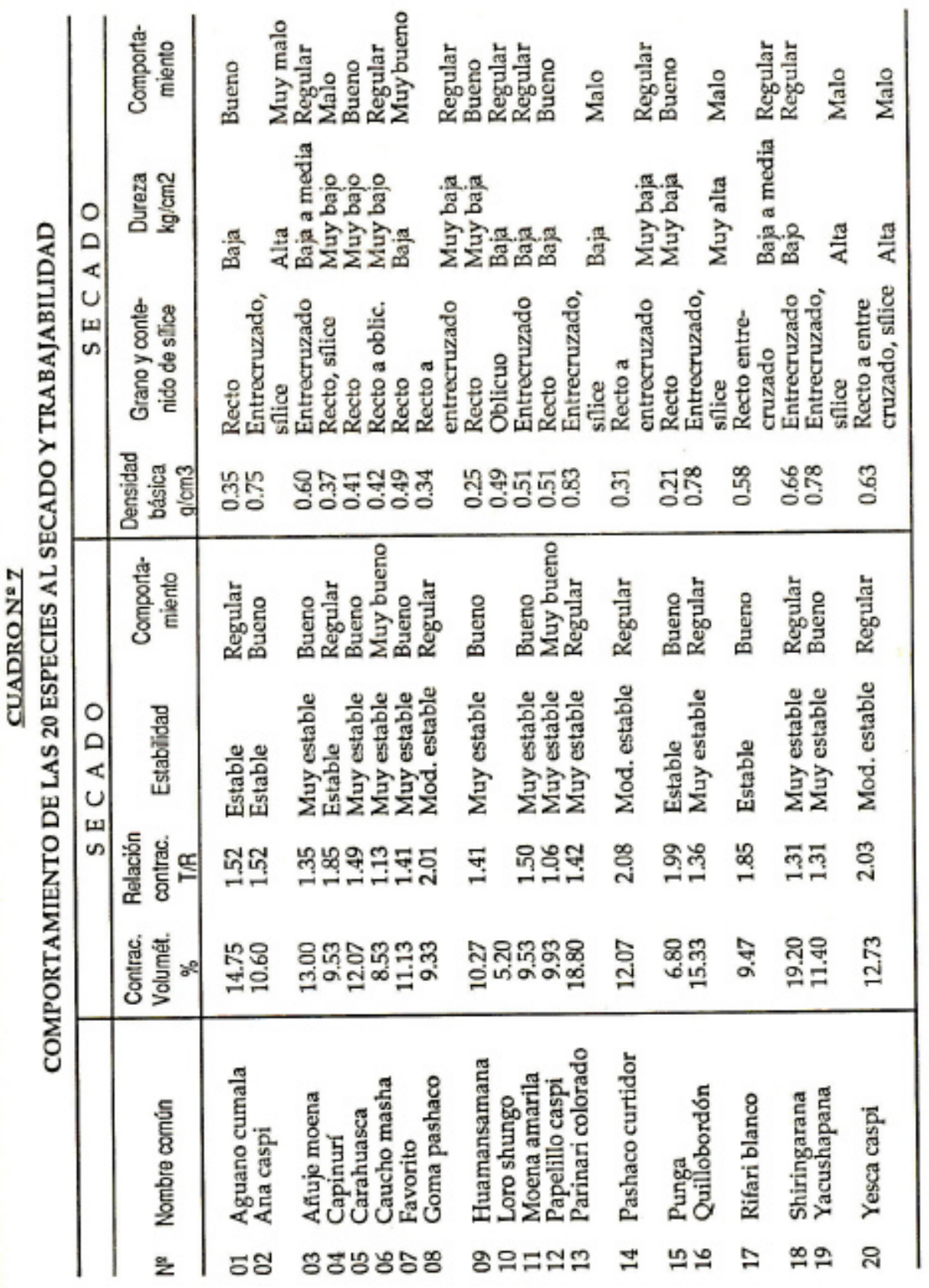




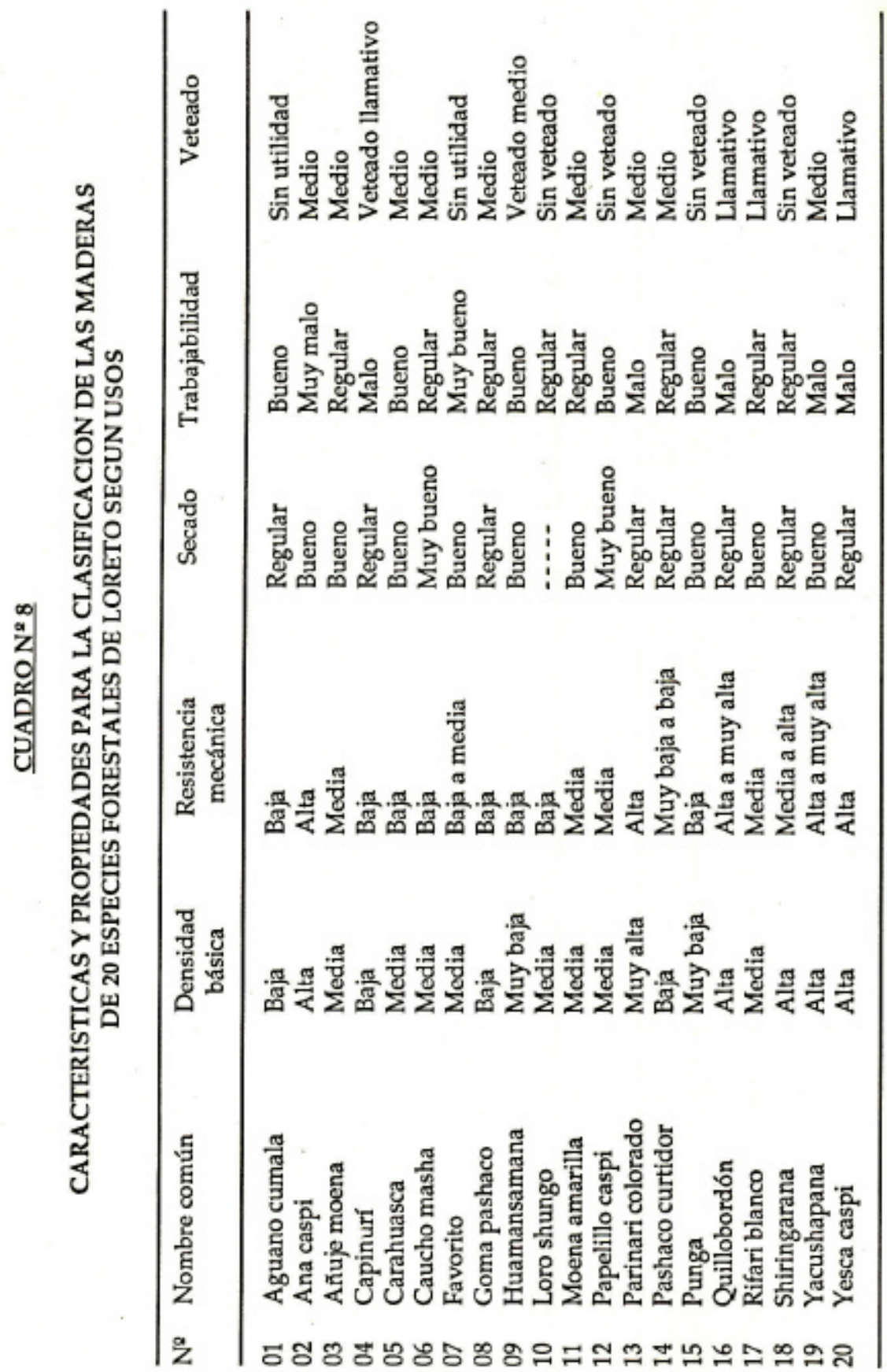




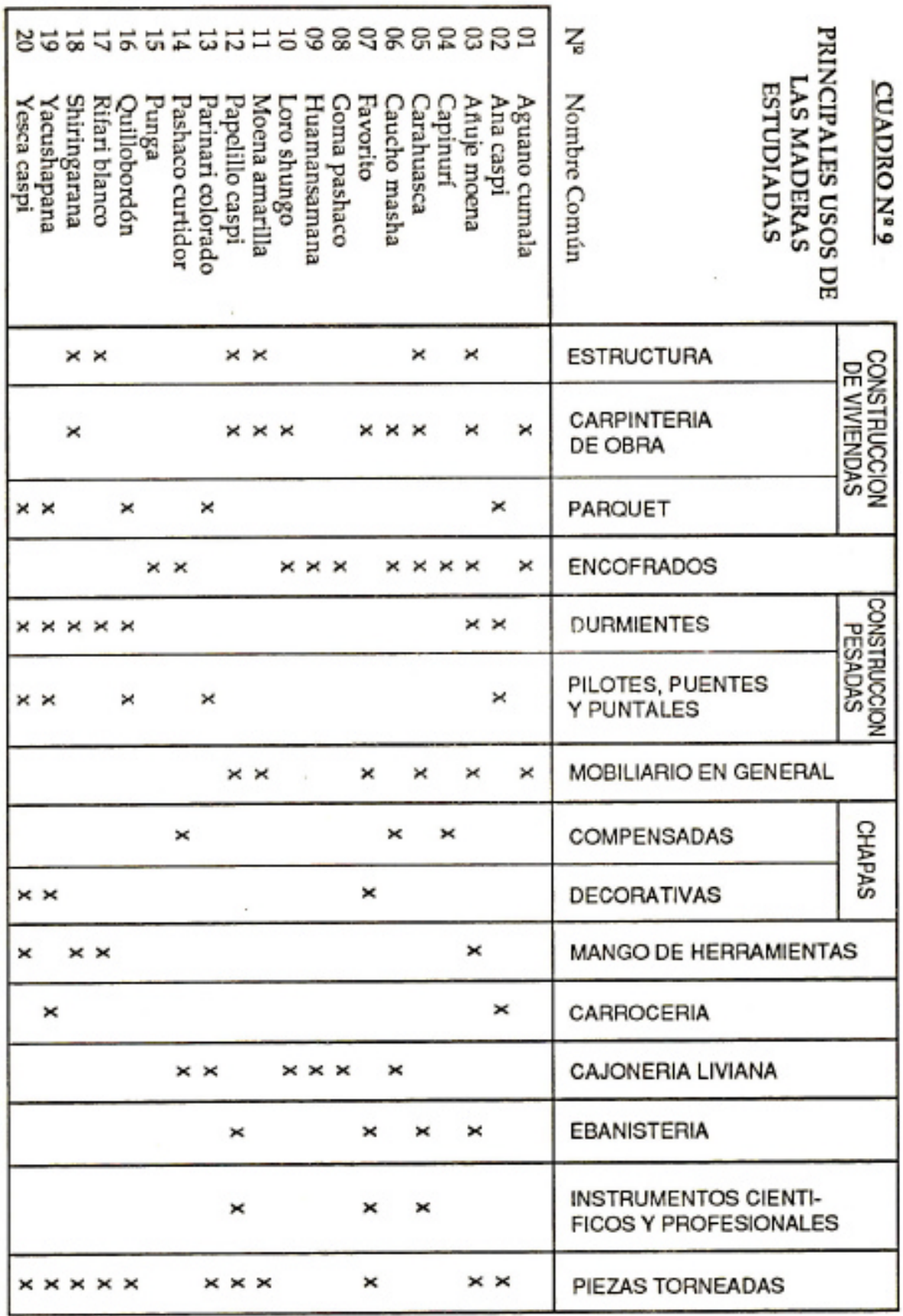

